\title{
Retrospective analysis of a dedicated care pathway for nonalcoholic fatty liver disease in an integrated US healthcare system demonstrates support of weight management and improved ALT
}

Heather Patton ${ }^{1,2^{*}}$ (D, Raoul Burchette ${ }^{3}$, Stephanie Tovar $^{3}$, Jose Pio ${ }^{3}$, Jiaxiao Shi ${ }^{3}$ and Lisa M. Nyberg ${ }^{1,3}$

\begin{abstract}
Background: A care pathway for nonalcoholic fatty liver disease (NAFLD) in Kaiser Permanente San Diego, California was instituted in August 2017 to improve efficiency of disease staging and promote lifestyle modification.

Methods: The NAFLD Care Pathway includes: (1) patient education (2) vibration controlled transient elastography (VCTE) examination (3) hepatology consultation for VCTE $\geq 8 \mathrm{kPa}$ and (4) referral to weight management (WM). Patients referred to the pathway during the first 6 months of its implementation were studied for adherence to its components and impact on weight change and ALT values in the 12 months following referral. Retrospective assessment of WM participation, change in weight, and change in ALT were evaluated in the 12-months following referral and compared to changes 12-months prior. Student's t-test or Wilcoxon signed rank test were used as appropriate $(p<0.05)$.

Results: 632 patients were included. 575 (91.0\%) completed VCTE examination with mean liver stiffness $8.5 \mathrm{kPa}$ (SD 9.2). 52 patients had mean liver stiffness $\geq 15 \mathrm{kPa}$. 180/632 (28.5\%) attended NAFLD education. 153/632 (24.2\%) were offered hepatology clinic and 136/153 (88.9\%) completed at least 1 appointment. Participation in WM was 24/632 (3.8\%) prior to referral and 67/632 (10.6\%) after referral and increased among patients who attended NAFLD education. Mean weight change following referral was $-0.69 \mathrm{~kg}$ (SD $6.58 \mathrm{~kg}$ ) among patients without WM and $-7.78 \mathrm{~kg}$ (SD $13.43 \mathrm{~kg}$ ) with WM. Overall, $44.2 \%$ of participants experienced weight gain after referral, $40.8 \%$ had weight loss $<5 \%$ and $15 \%$ had weight loss $\geq 5 \%$. Variables associated with weight loss included WM $(p<0.0001)$ and higher liver stiffness $(p=0.0066)$. Mean ALT change was -15.2 (SD 38.5) U/L without WM and - 28.8 (SD 29.6) U/L with WM.

Conclusions: A care pathway for NAFLD within a large, integrated healthcare system provides non-invasive disease staging and minimizes hepatology clinic utilization to those with more advanced disease. Referral was associated with increased enrollment in WM, weight loss, and decreased ALT. Given its impact on healthcare resources, strategies to improve NAFLD identification, staging, and promotion of lifestyle modification are imperative.
\end{abstract}

*Correspondence: Heather.Patton@VA.gov

1 Southern California Permanente Medical Group, Division

of Gastroenterology, Garfield Specialty Center, 5893 Copley Drive, San

Diego, CA 92111, USA

Full list of author information is available at the end of the article

(c) The Author(s) 2020. Open Access This article is licensed under a Creative Commons Attribution 4.0 International License, which permits use, sharing, adaptation, distribution and reproduction in any medium or format, as long as you give appropriate credit to the original author(s) and the source, provide a link to the Creative Commons licence, and indicate if changes were made. The images or other third party material in this article are included in the article's Creative Commons licence, unless indicated otherwise in a credit line to the material. If material is not included in the article's Creative Commons licence and your intended use is not permitted by statutory regulation or exceeds the permitted use, you will need to obtain permission directly from the copyright holder. To view a copy of this licence, visit http://creativecommons.org/licenses/by/4.0/. The Creative Commons Public Domain Dedication waiver (http://creativeco mmons.org/publicdomain/zero/1.0/) applies to the data made available in this article, unless otherwise stated in a credit line to the data. 
Keywords: Nonalcoholic fatty liver disease (NAFLD), Non-invasive fibrosis test, Clinical management, Weight loss

\section{Background}

Nonalcoholic Fatty Liver Disease (NAFLD) is a highly prevalent condition commonly seen in association with metabolic comorbidities such as obesity, diabetes mellitus (DM), and dyslipidemia [1-3]. NAFLD encompasses a spectrum of severity ranging from typically non-progressive simple steatosis or nonalcoholic fatty liver (NAFL) to nonalcoholic steatohepatitis (NASH) characterized by inflammation, hepatocellular ballooning injury, and progressive fibrosis [2]. Distinguishing NAFL from NASH in clinical practice can be difficult but is relevant given that the majority of liverrelated morbidity and mortality is seen in NASH and liver fibrosis is the most important variable predictive of prognosis [4]. For purposes of resource allocation, identification of patients with NASH serves to prioritize patients in greatest need of evaluation by gastroenterology and hepatology specialists [3].

Despite its high prevalence, there are significant knowledge gaps among primary providers in the diagnosis and management of NAFLD [5, 6]. The heavy clinical burden from NAFLD mandates partnership between primary care and specialty care with development of mutually agreeable triage processes to link high-risk patients with NAFLD to gastroenterology and hepatology providers. Optimal means of triage of NAFLD patients at the primary care setting should be performed with readily available clinical variables. Non-invasive testing, including both blood and imaging-based modalities, can be used for this purpose [7]. Additionally, the presence of Metabolic Syndrome (MetS) in NAFLD is associated with $\mathrm{NASH}$ and thus can help to target patients for additional assessment [8].

Kaiser Permanente (KP) is a large, integrated healthcare system with high penetrance in Southern California, serving approximately 650,000 members in San Diego. A dedicated care pathway for NAFLD in KP San Diego (KPSD) was implemented in 2017 with the goals of (1) assisting primary care providers (PCPs) in identifying patients with NAFLD most likely to have NASH and/or fibrosis (2) high throughput triage of NAFLD patients with noninvasive testing (3) provision of patient education regarding NAFLD diagnosis and (4) promotion of lifestyle modification programs through the healthcare plan aimed to promote weight loss. The primary aim of this study is to determine the initial success of this NAFLD care pathway in achieving these goals and the impact of this program on weight loss and improvement in liver blood tests.

\section{Methods}

A dedicated care pathway for NAFLD was implemented at KPSD in August 2017. Components of the care pathway include:

- Education of primary care providers (PCPs) regarding patient populations at risk for NAFLD and NASH, clinical evaluation for NAFLD, and associated cardiovascular risks in this population. This education was administered as a didactic session during a mandatory primary care Continuing Medical Education meeting. High-risk NAFLD patients were defined for PCPs as those with body mass index $(\mathrm{BMI}) \geq 30 \mathrm{~kg} / \mathrm{m}^{2}$, age $\geq 50$ years, and diabetes (DM).

- Automated approval of any Gastroenterology consultation placed with indication "Fatty Liver"

- All fatty liver referrals are offered vibration controlled transient elastography (VCTE, FibroS$\mathrm{can}^{\circledR}$ ) examination, education and referral to KPSD weight management (WM). FibroScan ${ }^{\circledR}$ examinations were performed by trained operators in the Gastroenterology Department and results were reviewed and interpreted by hepatology physicians.

- NAFLD Education was developed by a hepatologist (HP) and delivered by trained health educators (registered nurses) in a 90-min classroom session in the Health Education Department. The curriculum includes a definition of NAFLD and its spectrum, tests used in the diagnosis and staging of NAFLD, risk factors, potential health outcomes, and data regarding the efficacy of lifestyle modification in improving or reversing NAFLD. Information about WM programs offered by the healthcare plan are also reviewed during the education session.

- Patients with liver stiffness measurement $(\mathrm{LSM}) \geq 8 \mathrm{kPa}$ are contacted and offered a clinic visit with Hepatology. The threshold of $8 \mathrm{kPa}$ was selected as this would identify patients with an estimated liver fibrosis stage of 2 [9]. Patients with $\mathrm{LSM}<8 \mathrm{kPa}$ are informed that if they pursue lifestyle modification with modest weight reduction that fatty liver may be improved or reversed.

\section{Primary Objectives:}

- Improvement in liver blood tests (ALT) at 12 months following referral relative to change in ALT in the 12 months prior to referral (historical period). 
- Change in weight at 12 months following referral relative to change in weight in the historical period. Secondary Objectives:

- Enrollment in WM programs through KPSD.

- Performance of VCTE (FibroScan ${ }^{\circledR}$ ) as a triage measure for NAFLD (rate of exam completion, technical success for exams performed, and percentage patients identified with advanced liver fibrosis).

- Patient participation in NAFLD education.

- Utilization of hepatology clinic for NAFLD patients identified with $L S M \geq 8 \mathrm{kPa}$.

- Change in HgbA1C at 12 months following referral relative to change in $\mathrm{HgbA1C}$ in the historical period.

- Determination of factors associated with weight loss and change in ALT.

\section{Design}

This is a retrospective analysis of patients referred to the KPSD NAFLD Care Pathway during the first 6 months of its implementation. This study was approved by the KP Southern California (KPSC) Institutional Review Board prior to initiation and patients were de-identified for anal- registries. KP disease registries require a combination of ICD codes (2 or more), laboratory results (for diabetes) plus pharmacy dispensing records to identify patients. Dyslipidemia was identified through ICD codes (E78.00, E87.01, E78.1, E78.2, E78.5). Cardiovascular disease was defined according to a comprehensive set of ICD codes (available as supplemental data in Additional file 1). VCTE (FibroScan ${ }^{\circledR}$ ) reports were evaluated for date of exam, median liver stiffness measurement (LSM) and interquartile range \% (IQR\%). VCTE exams with IQR\% > 30 were deemed invalid and excluded from analysis. Hepatology utilization was assessed by documentation of any hepatology visit type (telephone or clinic) following referral among patients with $L S M \geq 8 \mathrm{kPa}$. Chart review was utilized to determine attendance at NAFLD class. Participation in WM programs included attendance at any of the programs offered through KPSD during the historical and study periods, including full and partial fasting programs (OPTIFAST ${ }^{\circledR}$ ) and Healthy Balance (16 weekly group sessions promoting plant-based nutrition and physical exercise).

NAFLD Fibrosis score (NFS) was calculated from data, where available, within 6 months of referral from the following formula [10]:

$$
\begin{aligned}
\text { NAFLD fibrosis score }= & -1.675+0.037 \times \text { age }(\text { years })+0.094 \times \operatorname{BMI}\left(\mathrm{kg} / \mathrm{m}^{2}\right) \\
& +1.13 \times \text { impaired fasting glucose } / \text { diabetes }(\text { yes }=1, \text { no }=0) \\
& +0.99 \times \text { AST } / \text { ALT ratio }-0.013 \times \text { platelet }\left(\times 10^{9} / \mathrm{L}\right) \\
& -0.66 \times \text { albumin }(\mathrm{g} / \mathrm{dL}) .
\end{aligned}
$$

ysis. Patients were identified for study through a clinical database maintained by hepatology case managers and included after verification that their referral occurred between August 1, 2017 and January 31, 2018 with an International Classification of Diseases (ICD) Code for NAFLD/NASH (K76.0, K75.81). Patients were excluded from study if there was evidence of Gastroenterology referral or specialty care outside of the study window, a gap in healthcare coverage exceeding 45 days during the study period, or an ICD code indicating an alcohol use disorder or alcoholic liver disease (K70.0, K70.30, K70.9, K77. $\mathrm{xx}, \mathrm{F} 10 . \mathrm{xx})$. For purposes of evaluating study end points, patient outcomes were assessed at 12 months following their referral date and compared to historical patient data assessed at 12 months prior to their referral date.

Baseline patient characteristics were determined at the time of the referral and included demographics, anthropometrics, comorbid medical conditions, and laboratory data.

Diabetes/prediabetes and hypertension (HTN) were determined from Southern California KP disease
NFS was categorized as low risk $(<-1.455$, high negative predictive value for advanced fibrosis), indeterminate risk $(-1.455$ to 0.676$)$ or high risk for advanced liver fibrosis $(>0.676$, high positive predictive value for advanced fibrosis). Weight change following referral to the NAFLD Care Pathway was categorized as: weight gain (any), $<5 \%$ weight loss, $5-10 \%$ weight loss or $\geq 10 \%$ weight loss. Change in ALT was assessed for each of these categories for those patients who had ALT data in the post intervention study period.

\section{Statistical analysis}

Change in body weight (kilograms), change in ALT (U/L), and change in HgbAlc (\%) were calculated during the historical period (from data closest to 12 months prior to referral to time of referral) and compared to changes in these variables during the study period (from time of referral to data available closest to 12 months from referral). Student's t-test or Wilcoxon signed rank test were used as appropriate (significance set at $p<0.05$ ).

Linear regression was performed to evaluate factors associated with weight loss and ALT improvement in the 
study population. Variables assessed included attendance at NAFLD education, having had a hepatology clinic visit, enrollment in weight management programs, LSM results, presence of comorbidities (DM, HTN, and dyslipidemia), and whether patients met definition for high risk NAFLD $\left(\mathrm{DM}+\right.$ age $\geq 50$ years $\left.+\mathrm{BMI} \geq 30 \mathrm{~kg} / \mathrm{m}^{2}\right)$. The covariates were examined by correlation for multicolinearity and other native associations. Ad hoc models of change in outcome between periods were built using both stepwise selection and backward elimination to verify that the model was reasonably stable if both methods produced the same results using successively stricter thresholds for entry and removal (both at 0.15 and then both at 0.05 ). We included covariates that were close to the final threshold.

\section{Results}

A total of 632 patients referred to the NAFLD Care Pathway during the initial 6 months of its implementation (August 1, 2017-January 31, 2018) met inclusion and exclusion criteria for study (Fig. 1). Patient characteristics are summarized in Table 1. At baseline (time of referral), the mean age was 53.7 years with female predominance ( $56.7 \%$ vs. $43.4 \%$ males). White race was most prevalent, followed by Hispanic, Asian and Black. Obesity prevalence was high, with a mean BMI of $34 \mathrm{~kg} / \mathrm{m}^{2}$. Comorbidities included in the Metabolic Syndrome were common (diabetes/prediabetes: 32.6\%, dyslipidemia: 59.7\%, and hypertension: $48.3 \%$ ). Nearly $10 \%$ of the population had a diagnostic code for cardiovascular disease. Liver biochemistries were mildly abnormal (mean ALT $59.4 \mathrm{U} / \mathrm{L}$, AST 41.8 U/L, GGT 78.3 U/L) while baseline albumin, INR and bilirubin were within normal range $(3.9 \mathrm{~g} / \mathrm{dL}$, 1.0 and $0.7 \mathrm{mg} / \mathrm{dL}$, respectively). Baseline platelet count was normal at $240.1\left(\times 10^{9} / \mathrm{L}\right)$.

Flow chart illustrating disposition of candidate patients initially identified through a clinical database maintained by case managers in hepatology. Candidate patients were restricted to those referred during the initial 6 months of the care pathway's implementation and then further subjected to exclusion criteria. A total of 632 patients were included in this analysis and adherence to aspects of the care pathway are shown.

\section{High-risk NAFLD}

A total of $101(16 \%)$ patients met pre-defined high-risk NAFLD criteria (age $\geq 50$ years, DM, and $B M I \geq 30 \mathrm{~kg} /$ $\left.\mathrm{m}^{2}\right)$. High-risk NAFLD patients $(\mathrm{n}=40$ with available labs) had mean ALT of $55.1 \mathrm{U} / \mathrm{L}$ (range 14-111, $\mathrm{SD}=21.6$ ) vs. $66.5 \mathrm{U} / \mathrm{L}$ (range $10-287, \mathrm{SD}=43.8$ ) for the remaining NAFLD patients $(\mathrm{n}=179$ with available labs) ( $p=0.295$ [based on chi-square for Wilcoxon ranksum statistic]). Data were available to calculate a NAFLD

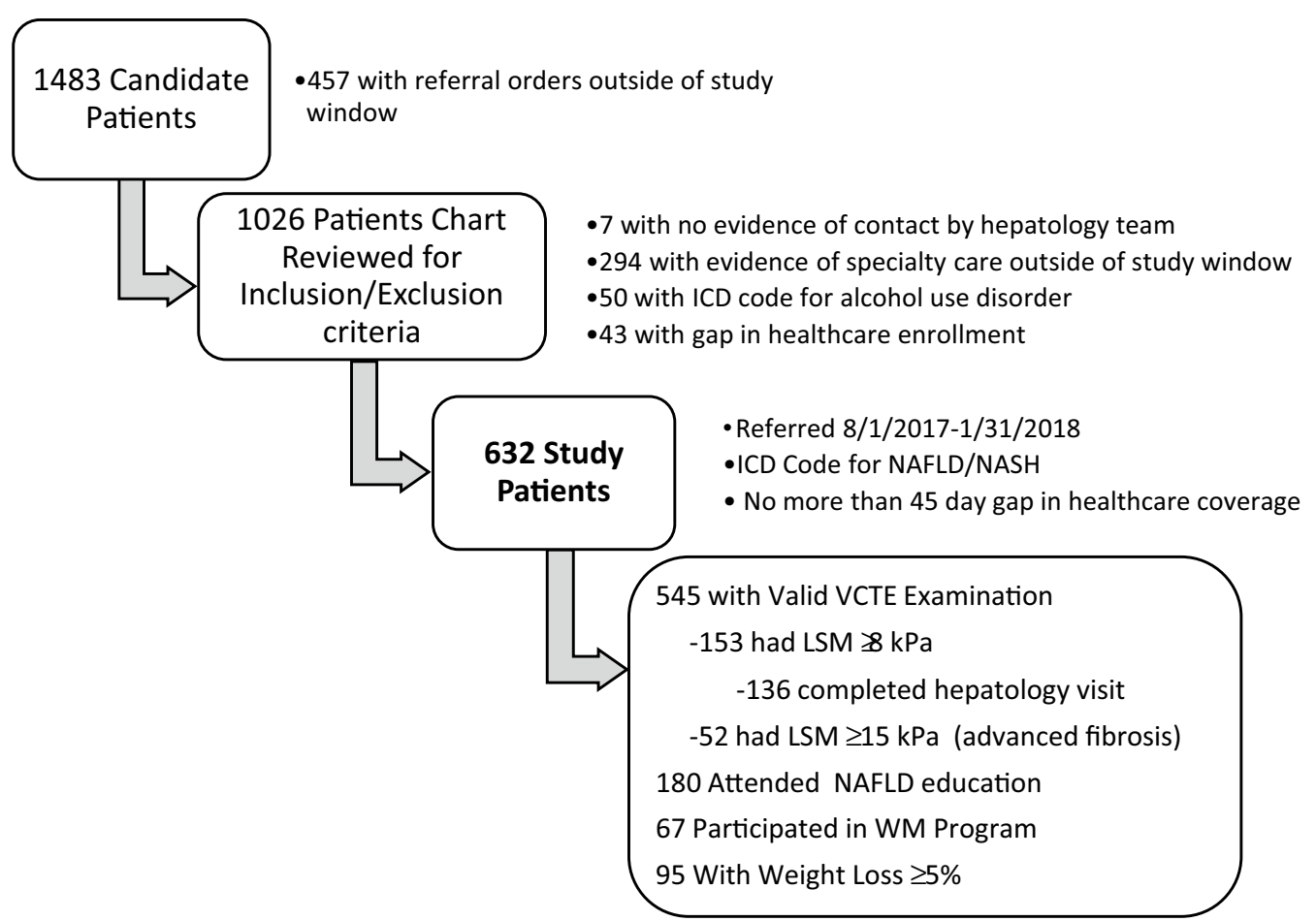

Fig. 1 Flow Chart of patients referred to NAFLD Care Pathway to arrive at study population 
Table 1 Baseline characteristics of patients referred to the NAFLD care pathway 8/1/2017-1/31/2018

\begin{tabular}{|c|c|}
\hline Population characteristic & Total \\
\hline $\begin{array}{l}\text { Study population referred to NAFLD Care Pathway } \\
8 / 1 / 2017-1 / 31 / 2018\end{array}$ & 632 \\
\hline \multicolumn{2}{|l|}{ Gender, N (\%) } \\
\hline Male & $274(43.4 \%)$ \\
\hline Female & $358(56.7 \%)$ \\
\hline Mean age (SD) years & $53.7(13.0)$ \\
\hline \multicolumn{2}{|l|}{ Race/ethnicity, N (\%) } \\
\hline White & $271(42.9 \%)$ \\
\hline Hispanic & $247(39.1 \%)$ \\
\hline Asian & $66(10.4)$ \\
\hline Black & $20(3.2 \%)$ \\
\hline Other/unknown/multiple & $28(4.4 \%)$ \\
\hline Mean BMI (SD) kg/m² & $34.0(6.8)$ \\
\hline Diabetes/Prediabetes, N (\%) & $206(32.6 \%)$ \\
\hline Dyslipidemia, N (\%) & $377(59.7 \%)$ \\
\hline HTN, N (\%) & $305(48.3 \%)$ \\
\hline Cardiovascular Disease, N (\%) & $62(9.8 \%)$ \\
\hline ALT U/L, mean (SD) $(N=602)$ & $59.4(42.9)$ \\
\hline AST U/L, mean $(S D)(N=405)$ & $41.8(36.4)$ \\
\hline GGT U/L, mean $(S D)(N=384)$ & $78.3(104.2)$ \\
\hline Albumin g/dL, mean (SD) $(N=198)$ & $3.9(0.3)$ \\
\hline Total Bilirubin mg/dL, mean (SD) $(\mathrm{N}=520)$ & $0.7(0.4)$ \\
\hline Platelet count $\left(\times 10^{9} / \mathrm{L}\right)$ mean $(\mathrm{SD})(\mathrm{N}=517)$ & $240.1(72.0)$ \\
\hline INR, mean $(S D)(N=171)$ & $1.0(0.3)$ \\
\hline Ferritin $\mathrm{ng} / \mathrm{mL}$, mean $(\mathrm{SD})(\mathrm{N}=228)$ & $239.9(255.5)$ \\
\hline HgbA1c \%, mean (SD) $(\mathrm{N}=556)$ & $6.3(1.2)$ \\
\hline Total Cholesterol mg/dL, mean (SD) $(\mathrm{N}=479)$ & $182.7(44.6)$ \\
\hline $\mathrm{LDL} \mathrm{mg} / \mathrm{dL}$, mean $(\mathrm{SD})(\mathrm{N}=467)$ & $107.2(36.9)$ \\
\hline $\mathrm{HDL} \mathrm{mg} / \mathrm{dL}$, mean $(\mathrm{SD})(\mathrm{N}=479)$ & $45.4(11.5)$ \\
\hline Triglycerides mg/dL, mean (SD) $(\mathrm{N}=329)$ & $176.8(253.8)$ \\
\hline
\end{tabular}

Data shown for the initial cohort of patients referred to the NAFLD Care Pathway upon its creation in August 2017. All data shown are from the time of referral. Laboratory values of interest were not available in all participants in this retrospective analysis and thus numbers for which individual results are available are shown in parentheses. Comorbid health conditions were identified through ICD codes and KP disease registries

Fibrosis Score in 154/632, most of whom (80.6\%) were in the low or indeterminate risk categories (Fig. 2).

Data shown for 154 patients with available labs to calculate a NAFLD Fibrosis Score at the time of referral. Results of the NAFLD Fibrosis Score are categorized as low risk $(<-1.455)$, indeterminate $(-1.455$ to 0.675$)$, and high risk (>0.675) and reported as percent of the 154 with available data within each category.

\section{VCTE results}

575 (91.0\%) patients completed VCTE (FibroScan ${ }^{\circledR}$ ) examination and 545 (94.8\%) exams were deemed reliable $(\mathrm{IQR} \leq 30 \%)$. Mean LSM results for the entire study population was $8.5 \mathrm{kPa}$ (SD 9.2). Most patients $(286 / 545=52.5 \%)$ had normal liver stiffness measurement $(<6 \mathrm{kPa})$ (Fig. 3). 392 patients $(71.9 \%)$ had $\mathrm{LSM}<8 \mathrm{kPa}$ and thus were not offered an appointment in hepatology while 153 patients (28.1\%) had LSM results $\geq 8 \mathrm{kPa}$ and were offered an appointment in hepatology clinic. 52 patients (9.5\%) had LSM $\geq 15 \mathrm{kPa}$ indicating possible advanced liver fibrosis/cirrhosis. Median LSM was $7.4 \mathrm{kPa}$ (range $2.2-70.7, \mathrm{SD}=11.84$ ) for highrisk NAFLD vs. $5.6 \mathrm{kPa}$ (range $2.2-75.0, \mathrm{SD}=8.93$ ) for the remaining NAFLD patients $(p<0.001$ [based on chisquare for Wilcoxon rank-sum statistic]).

Results of 545 VCTE (FibroScan ${ }^{\circledR}$ ) examinations performed in 575 patients referred to the NAFLD Care Pathway with IQR $\leq 30 \%$. Data shown are for the percent of these 545 examinations with median liver stiffness measurement in categories of increasing severity.

\section{NAFLD education}

A total of $180 / 632(28.5 \%)$ patients attended the single NAFLD education session that was offered to all patients.

\section{Hepatology clinic utilization}

A total of 153/632 (24.2\%) patients were offered a hepatology clinic visit based on their VCTE examination results (LSM $\geq 8 \mathrm{kPa}) .136$ of 153 (88.9\%) patients offered appointments completed at least 1 hepatology clinic visit within 1 year of referral (representing $21.5 \%$ of the study population). There were a total of 188 visits completed by the 136 patients with 25 patients completing at least 1 telephone visit and 126 patients completing at least 1 office visit.

\section{WM program}

Participation in a KP WM program was $24 / 632(3.8 \%)$ in the 12 months prior to referral and 67/632 (10.6\%) after referral. Participation in WM after referral was greater (14.4\%) among those who attended NAFLD Class versus those who did not $(9.1 \%)(p=0.048)$ (Table 2). There was no association between NAFLD class attendance and participation in WM programs prior to referral to the NAFLD Care Pathway $(p=0.70)$.

Patient participation in any of the KP Weight Management Programs in the 12 months following referral to the NAFLD Care Pathway shown according to whether patients attended the single NAFLD Education session offered to all patients. Weight management program participation was higher in the subset of patients who had attended NAFLD Class $(p=0.048)$.

\section{Change in weight}

In the 12 months prior to referral, mean weight change was $+0.09 \mathrm{~kg}$ (SD $4.85 \mathrm{~kg})$. Mean weight change following referral was $-1.45 \mathrm{~kg}(\mathrm{SD} 7.94 \mathrm{~kg})$ overall, 


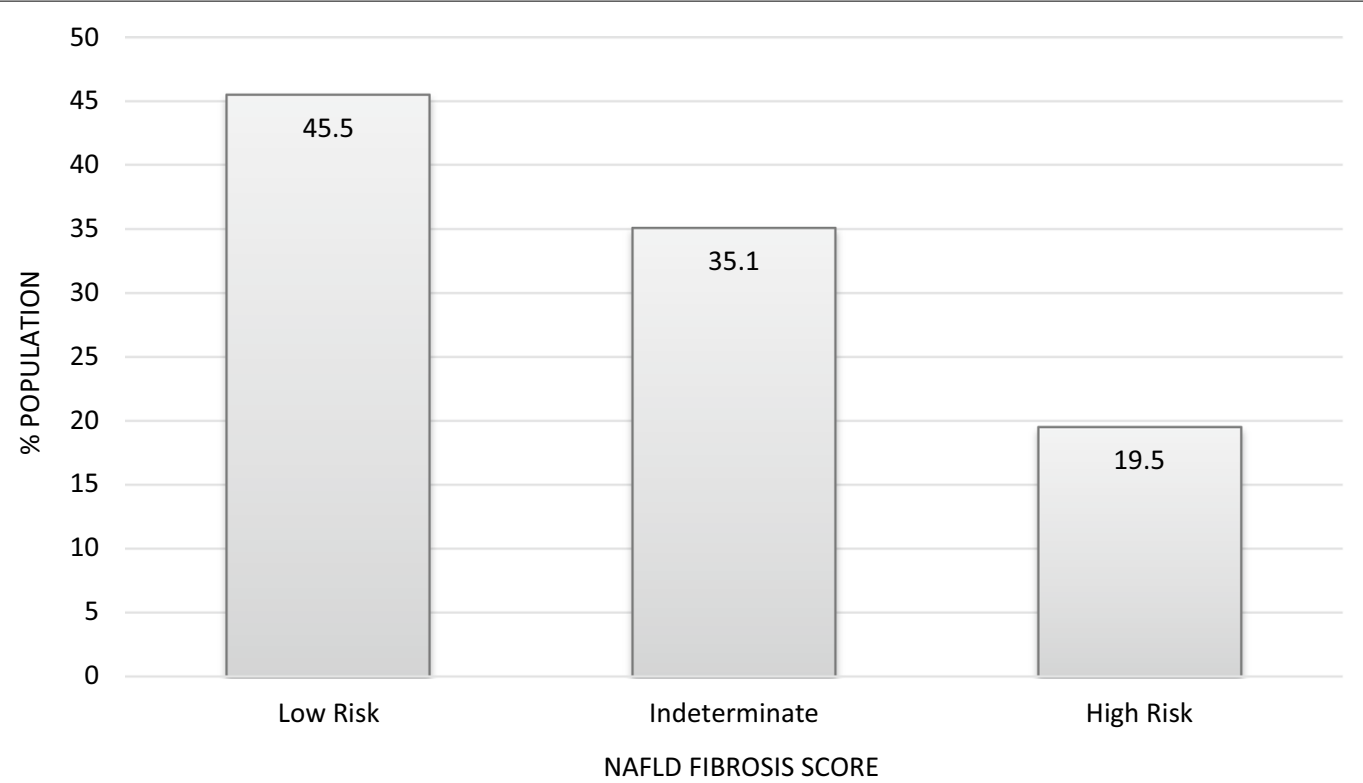

Fig. 2 NAFLD Fibrosis score results at time of referral

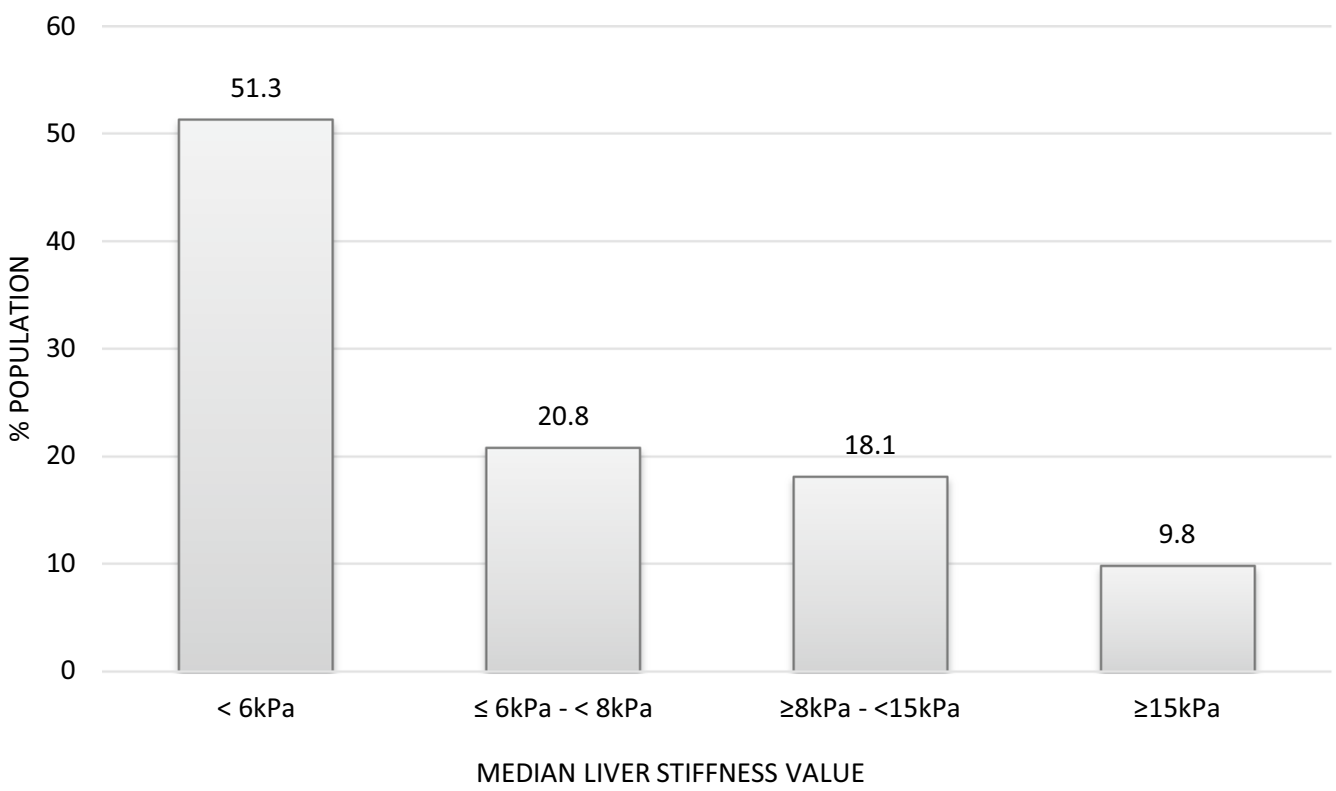

Fig. 3 Breakdown of liver stiffness measurements on VCTE examination

$-0.68 \mathrm{~kg}$ (SD $6.58 \mathrm{~kg}$ ) among patients who did not enroll in WM programs and $-7.80 \mathrm{~kg}$ (SD $13.43 \mathrm{~kg}$ ) with WM program participation. While over $50 \%$ of patients achieved some weight loss following referral, only a minority of patients achieved weight loss in excess of 5\% (Fig. 4). Change in mean ALT (standard deviation) was determined according to weight change category in the post referral period: $-11.4 \mathrm{U} / \mathrm{L}$ (35.7) in 83 patients with weight gain (any), $-16.3 \mathrm{U} / \mathrm{L}$ (27.1) in 72 patients with $<5 \%$ weight loss, $-22.0 \mathrm{U} / \mathrm{L}(40.3)$ in 28 patients with $5-10 \%$ weight loss, and $-25.6 \mathrm{U} / \mathrm{L}$ (39.3) in 25 patients with $\geq 10 \%$ weight loss (Fig. 4). On linear regression analysis, the variables associated with weight loss after referral included participation in WM 
Table 2 Relationship between weight management program and NAFLD class participation

\begin{tabular}{llcr}
\hline $\begin{array}{l}\text { NAFLD class } \\
\text { attendance }\end{array}$ & \multicolumn{2}{l}{$\begin{array}{l}\text { Weight management program } \\
\text { participation following referral }\end{array}$} & $\%$ \\
\cline { 2 - 3 } & No & Yes & \\
\hline No & 411 & 41 & 9.1 \\
Yes & 154 & 26 & 14.4 \\
\hline
\end{tabular}

program $(p<0.0001)$ and higher LSM result on VCTE examination $(p=0.007)$.

The bar graph depicts percent of patients in each category of weight change among 632 patients referred to the NAFLD Care Pathway in the 12 months following the referral date. Overlying this, the line graft depicts the mean change in ALT [ $\triangle \mathrm{ALT}=$ (ALT value 12 months after referral)-(ALT value at time of referral)] for patients within each weight change category. As percentage weight loss increased, the average decline in ALT value during this time interval was also increased.

\section{Change in ALT}

The average change in ALT among those who had values in both periods $(\mathrm{n}=219)$ was $-3.1 \mathrm{U} / \mathrm{L}(\mathrm{SD} 43.6)$ prior to referral and $-15.4 \mathrm{U} / \mathrm{L}$ (SD 35.2) following referral. Mean ALT change over the entire study period was - 17.1 (SD 38.1) U/L overall, - 15.2 (SD 38.5) U/L without WM and -28.8 (SD 29.6) U/L with WM. On linear regression analysis, no significant predictors of decrease in ALT were identified aside from higher baseline ALT value.

Change in ALT after the index date (following referral to the care pathway) based on NAFLD class attendance and WM participation was evaluated in the 219 patients with ALT results available throughout the study period. The 82 patients who took NAFLD Class had a reduction in ALT during the follow-up period of 17.1 $\mathrm{U} / \mathrm{L}$ compared to a reduction in ALT of $14.5 \mathrm{U} / \mathrm{L}$ in the 137 patients who did not take NAFLD class $(p=0.11$, Wilcoxon rank sum comparison). Change in ALT in the follow-up period among the 38 patients who enrolled in WM was a decrease of $25.7 \mathrm{U} / \mathrm{L}$ compared to a decrease of $13.3 \mathrm{U} / \mathrm{L}$ in the 181 patients who did not enroll in WM ( $p=0.03$ for the Wilcoxon rank sum comparison).

\section{Change in HgbA1c}

A total of 310 patients had HgbA1c results available in both the pre- and post-referral periods. The change in HgbA1c among those who had values in both periods was a decrease from an average of $6.5 \%$ before referral to an average of $6.2 \%$ after referral ( $p=\mathrm{NS}$ ).

\section{Discussion}

Given the high prevalence of NAFLD with estimated burden in the US approaching 100 million, specialty evaluation of all patients is untenable [11]. The optimal setting, modality, and timing of triage for specialty referral remains to be determined (and may vary according to healthcare system, driven by factors such as patient

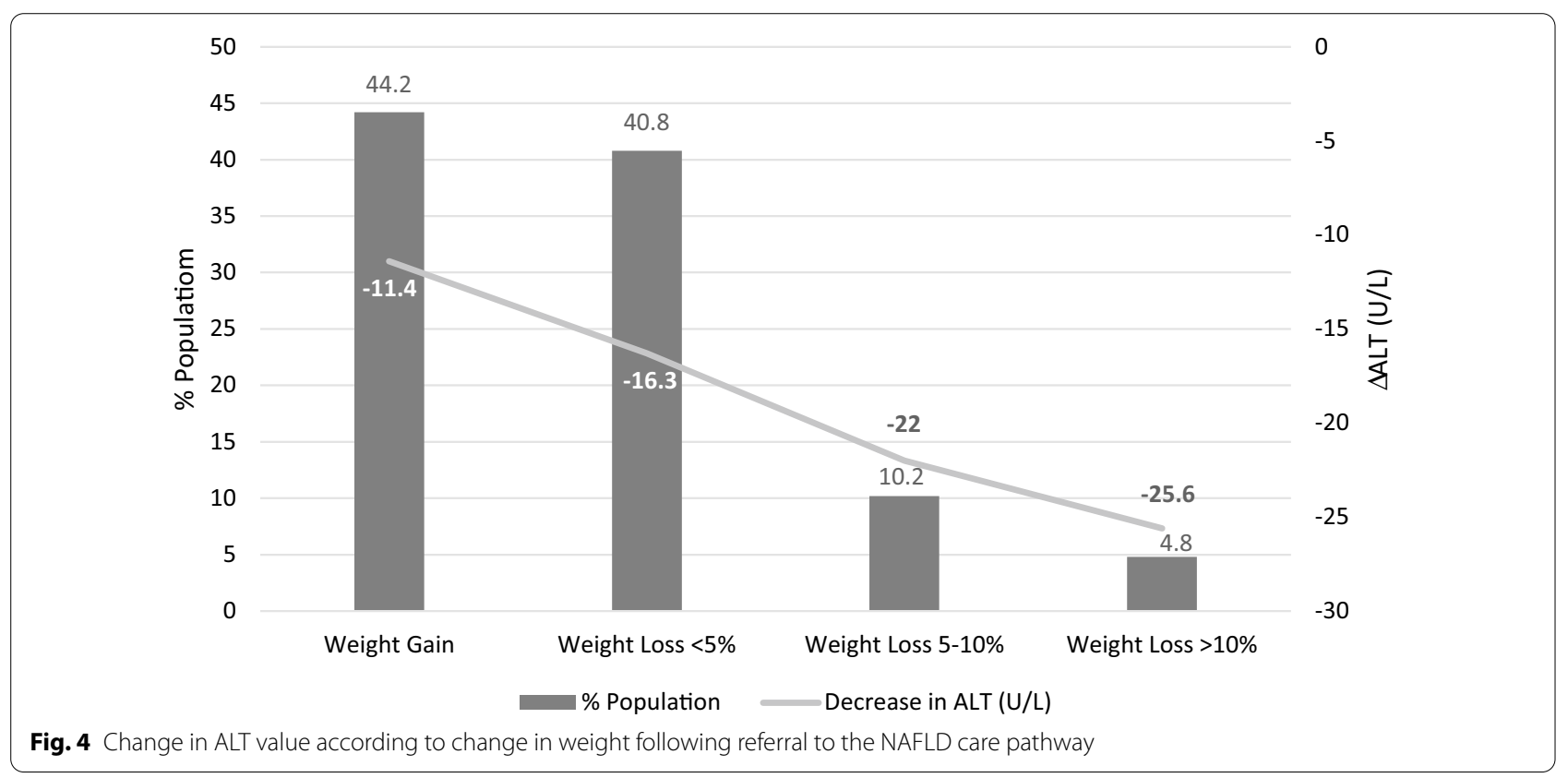


population characteristics and available resources). We have demonstrated the feasibility and clinical benefits of implementation of a dedicated care pathway for NAFLD within a large, integrated healthcare system in the US. Our evaluation of over 600 patients referred in the initial six months of this program suggests that primary care providers will utilize available resources for their patients with NAFLD, that patients with NAFLD are willing to pursue education and additional noninvasive testing (NIT) for their diagnosis, that patient education increases enrollment in weight management programs, and that weight management programs improve weight reduction and decrease in ALT.

The risk for liver-related mortality in NAFLD increases exponentially with stage of liver fibrosis, highlighting the importance of fibrosis assessment in clinical practice [4]. The American Association for the Study of Liver Disease (AASLD) Practice Guidelines support use of NIT for evaluating patients with NAFLD and to help guide identification of patients who may be appropriate for liver biopsy [2]. NAFLD Fibrosis Score and FIB4 both perform well in identifying patients with NAFLD who have advanced liver fibrosis [12]. Furthermore, high NFS score has been identified as a marker of increased mortality (pooled RR 4.54, 95\% CI 1.85-11.17) thus either or both of these readily available and inexpensive scoring systems are helpful tools in staging and prognosis of patients with NAFLD [13]. Calculation of NAFLD Fibrosis Score or FIB4 were not included in the initial KPSD NAFLD Care Pathway algorithm, but all patients were offered staging through VCTE. Patient adherence to this examination was excellent (91\%) and this approach identified 153 patients with increased likelihood of significant liver fibrosis, including 53 with $\mathrm{LSM} \geq 15 \mathrm{kPa}$ indicating high positive predictive value for cirrhosis [9]. Higher LSM in this study population was associated with weight loss subsequent to referral. Potential explanations for this observation include loss of fat and muscle stores in patients with advanced liver disease or the possibility that the communication of disease staging results may help to motivate patients in adopting lifestyle modification.

The NAFLD care pathway developed for KPSD minimized utilization of subspecialty providers to 152/632 (24.1\%) of patients with potentially advanced disease thus having a favorable impact on access to hepatology care. Improved recognition of NAFLD populations at risk for advanced disease may help to identify patients in the precirrhotic and early cirrhotic stages when effective therapy may help to halt or reverse disease progression and associated healthcare burdens. NAFLD may not be recognized or addressed prior to development of overt cirrhosis, often identified as an incidental finding in which case concomitant hepatocellular carcinoma (HCC) may be present [14]. In fact, NAFLD is emerging as the most common etiology of HCC $[15,16]$. Early identification of NAFLD patients with advanced fibrosis provides opportunity to implement HCC surveillance examinations, addressing data in the US that indicate HCC screening is performed less often in NAFLD cirrhosis and the diagnoses of HCC in NAFLD-related liver disease tends to occur later with more advanced cancers than other liver disease etiologies [17-19].

Our results demonstrate that PCPs are not intuitively referring high risk NAFLD patients for care as demonstrated by the low percentage of patients who met pre-defined high-risk criteria (16\%) and high risk for advanced fibrosis by NAFLD Fibrosis Score (19\%). As specialists, we need to partner with referring providers to ensure that they have the appropriate knowledge of NAFLD patients in high risk categories, are comfortable with clinical tools for risk assessment such as FIB4 and NAFLD Fibrosis Score and have access to resources to support patients in their efforts at lifestyle modification [6]. In KP Southern California, the electronic medical record (EMR) now has an embedded NAFLD Fibrosis Score calculator that conveys results with interpretation. Such automated tools, particularly when incorporated into the EMR, offer the best opportunity to support overburdened practitioners in assessing NAFLD. Primary care-based assessment with NIT, including laboratorybased scores and VCTE, has been shown to be cost effective and to markedly reduce referral for specialty care $[20,21]$. Future iterations of this KPSD NAFLD Care Pathway should incorporate NIT at the level of the referring provider to further optimize resource utilization and improve the financial sustainability of this program.

While there are several pharmacotherapies under investigation for the treatment of NASH, lifestyle modification remains the standard of care for management of NAFLD [2, 22]. Nutrition, exercise, and weight loss are effective at improving NASH but are difficult to institute and sustain in clinical practice [23, 24]. Exercise alone or combined with dietary intervention, even absent weight loss, may reduce intrahepatic fat content [25]. A 5-10\% weight loss via dietary intervention in combination with exercise is recommended for management of NAFLD yet resources to support these behaviors are rarely available outside of the context of a clinical trial and success is likely hampered by a lack of clinical training in effective behavior change interventions [24, 26]. Data from the National Health and Nutrition Examination Survey (NHANES) indicate that physician counseling for weight loss among patients with NAFLD occurs at low rates (46\%). While this counseling is associated with patient reported effort to lose weight, it was not demonstrated to improve rates of significant $(\geq 5 \%)$ weight reduction 
[27]. Our real-world experience in a large-scale program to encourage lifestyle modification for NAFLD demonstrated a nearly threefold increase in WM program participation (10.6\% versus $3.8 \%)$ with greater enrollment in WM programs among those who had attended NAFLD education. Not surprisingly, participation in WM programs was associated with greater weight loss, as was a higher LSM result on VCTE exam. Furthermore, $10 \%$ of patients referred to this care pathway achieved a weight loss in the $5-10 \%$ range and nearly $5 \%$ of patients achieved a weight loss $\geq 10 \%$.

This study has several important limitations to acknowledge. As a retrospective analysis, variables of interest were not available for the entire study population at all time points of interest and ICD codes were used for the exclusion of alcohol related liver disease. While we were able to capture enrollment in WM programs offered through the healthcare plan, we were not able to account for participation in other formal or self-directed weight loss programs. Likewise, exercise is an important aspect of lifestyle modification for NAFLD with data supporting improved liver fat content, particularly when accompanied by weight loss [28]. While the benefits of exercise in management of NAFLD were included in NAFLD patient education, there was no routine assessment for physical activity level to determine if this parameter was impacted by the intervention. We also acknowledge that the duration of follow-up was relatively short such that the full benefits of the program as well as the durability of the intervention were not able to be assessed (both key in evaluating the sustainability of this intervention). Finally, these results may not be generalizable to healthcare systems that are not fully integrated and/or do not have internal resources such as weight management programs and a health education department to support this type of care delivery. The nature of healthcare in the US (lack of national healthcare or universal insurance coverage) results in tremendous heterogeneity in the approach to patient care for conditions like NAFLD and discrepancy in access to resources for weight management. Nearly $10 \%$ of national healthcare expenditure in the US is spent on prescription drugs, thus healthcare delivery systems may see favorable economic outcomes from the successful implementation of lifestyle modification programs in offsetting pharmaceutical utilization. Furthermore, particularly as therapies for NASH progress, payors may be compelled to support NIT programs for NAFLD to improve earlier identification of patients and provide opportunity to disrupt progression to advanced liver disease and its associated clinical and economic burdens [29].

Strengths of our study include our large patient population with greater than 600 individuals meeting study inclusion in the first six months of program implementation.

Despite the retrospective nature of this study, the large study population and robust tools available within this integrated healthcare system facilitated characterization of the study population. This study presents a unique, real world experience with implementation of systems to promote non-invasive testing, patient education/motivation, and lifestyle modification that offers both proof of concept as well as important lessons to modify future care pathways to optimal effect. While few patients achieved weight loss in excess of $5 \%$, the fact that $>50 \%$ of patients lost some weight in the year following referral is encouraging. The ALT value decreased over the course of the study period and the decline in ALT was greater in those with weight reduction. We also captured very high adherence to disease staging with VCTE examination, identifying nearly $10 \%$ of the study population with potentially advanced liver fibrosis/cirrhosis.

\section{Conclusions}

In conclusion, we have demonstrated the feasibility of implementing a dedicated care pathway for patients with NAFLD within a large, integrated healthcare system in the United States. This program was able to identify patients with liver fibrosis though VCTE examination and, in so doing, limit the impact of NAFLD on hepatology clinic utilization to those with evidence of advanced disease. Aided by patient education offered outside of the context of a clinic visit, this care pathway was successful in increasing enrollment in WM programs with over half of the patients achieving some weight loss in the 12 months following their referral. Liver blood tests (ALT) improved over the course of the study period, particularly among those with weight loss. Given the high prevalence of NAFLD, we believe that the implementation of care pathways within healthcare systems offer the opportunity to link patients with advanced fibrosis to specialty care prior to the onset of overt cirrhosis and to improve adherence to lifestyle modification.

\section{Supplementary information}

Supplementary information accompanies this paper at https://doi. org/10.1186/s12876-020-01492-9.

Additional file 1. Comprehensive list of ICD9 and ICD 10 Codes used to identify atherosclerotic cardiovascular disease in study population as a comorbid health condition.

\section{Abbreviations}

NAFLD: Nonalcoholic fatty liver disease; KP: Kaiser Permanente; VCTE: Vibration controlled transient elastography; WM: Weight management; NAFL:

Nonalcoholic fatty liver; NASH: Nonalcoholic steatohepatitis; MetS: Metabolic Syndrome; KPSD: KP San Diego; PCPs: Primary care providers; BMI: Body mass index; DM: Diabetes mellitus; LSM: Liver stiffness measurement; ICD: 
International Classification of Diseases; HTN: Hypertension; IQR\%: Interquartile range \%; NFS: NAFLD fibrosis score; NIT: Noninvasive testing; AASLD: American Association for the Study of Liver Disease; HCC: Hepatocellular carcinoma; EMR: Electronic medical record; NHANES: National Health and Nutrition Examination Survey.

\section{Acknowledgements}

Not applicable.

\section{Authors' contributions}

HP: study concept and design, writing of the draft manuscript; RB: statistical analysis methodology, data collection, data analysis; ST: oversight of protocol adherence, management of IRB submission and maintenance; JP: data collection, data analysis; JS: oversight of protocol adherence, statistical analysis methodology; LN: study co-design, manuscript revision. All authors read and approved the final manuscript.

\section{Funding}

The research is supported by a grant from the Regional Research Committee of Kaiser Permanente Southern California. Grant No. KP-RRC-20180503. Grant funds provided salary support for Department of Research and Evaluation staff for time spent dedicated to work on this research protocol.

\section{Availability of data and materials}

Anonymized data that support the findings of this study are made available from the corresponding author upon reasonable request from qualified researchers with documented evidence of training in human subject protections.

\section{Ethics approval and consent to participate}

This study protocol was adherent to the Declaration of Helsinki and was approved by the KP Southern California (KPSC) Institutional Review Board (IRB) prior to initiation. Informed consent for study participation was waived by the KPSC IRB.

\section{Consent for publication}

Not applicable.

\section{Competing interests}

The authors declare that they have no competing interests.

\begin{abstract}
Author details
1 Southern California Permanente Medical Group, Division of Gastroenterology, Garfield Specialty Center, 5893 Copley Drive, San Diego, CA 92111, USA. ${ }^{2}$ Section of Gastroenterology, VA San Diego Healthcare System, 3350 La Jolla Village Drive, San Diego, CA 92161, USA. ${ }^{3}$ Department of Research and Evaluation, Kaiser Permanente Research, Southern California Permanente Medical Group, 100 S. Los Robles Ave., Pasadena, CA 91101, USA.
\end{abstract}

Received: 4 March 2020 Accepted: 8 October 2020

Published online: 31 October 2020

\section{References}

1. Younossi Z, Tacke F, Arrese M, Chander Sharma B, Mostafa I, Bugianesi E, et al. Global perspectives on nonalcoholic fatty liver disease and nonalcoholic steatohepatitis. Hepatology. 2019;69(6):2672-82. https://doi. org/10.1002/hep.30251.

2. Chalasani N, Younossi Z, Lavine JE, Charlton M, Cusi K, Rinella M, et al. The diagnosis and management of nonalcoholic fatty liver disease: practice guidance from the American Association for the Study of Liver Diseases. Hepatology. 2018;67(1):328-57. https://doi.org/10.1002/hep.29367.

3. Younossi ZM, Blissett D, Blissett R, Henry L, Stepanova M, Younossi Y, et al. The economic and clinical burden of nonalcoholic fatty liver disease in the United States and Europe. Hepatology. 2016;64(5):1577-86. https:// doi.org/10.1002/hep.28785.

4. Dulai PS, Singh S, Patel J, Soni M, Prokop LJ, Younossi Z, et al. Increased risk of mortality by fibrosis stage in nonalcoholic fatty liver disease: systematic review and meta-analysis. Hepatology. 2017;65(5):1557-65. https://doi.org/10.1002/hep.29085.

5. Blais P, Husain N, Kramer JR, Kowalkowski M, El-Serag H, Kanwal F. Nonalcoholic fatty liver disease is underrecognized in the primary care setting. Am J Gastroenterol. 2015;110(1):10-4. https://doi.org/10.1038/ ajg.2014.134.

6. Abenavoli L, Procopio AC, Medić-Stojanoska M, Luzza F. Non-alcoholic fatty liver disease and primary care physicians. Minerva gastroenterologica e dietologica. 2020;66(1):4-5. https://doi.org/10.23736/S1121 $-421 \times .20 .02666-5$

7. Tapper EB, Lok ASF. Use of liver imaging and biopsy in clinical practice. $N$ Engl J Med. 2017. https://doi.org/10.1056/NEJMra1610570.

8. Sayiner M, Koenig A, Henry L, Younossi ZM. Epidemiology of nonalcoholic fatty liver disease and nonalcoholic steatohepatitis in the United States and the rest of the world. Clin Liver Dis. 2016;20(2):205-14. https://doi. org/10.1016/j.cld.2015.10.001.

9. Eddowes PJ, Sasso M, Allison M, Tsochatzis E, Anstee QM, Sheridan D, et al. Accuracy of FibroScan controlled attenuation parameter and liver stiffness measurement in assessing steatosis and fibrosis in patients with nonalcoholic fatty liver disease. Gastroenterology. 2019;156(6):1717-30. https://doi.org/10.1053/j.gastro.2019.01.042.

10. Angulo P, Hui JM, Marchesini G, Bugianesi E, George J, Farrell GC, et al. The NAFLD fibrosis score: A noninvasive system that identifies liver fibrosis in patients with NAFLD. Hepatology. 2007. https://doi.org/10.1002/ hep.21496.

11. Estes C, Razavi H, Loomba R, Younossi Z, Sanyal AJ. Modeling the epidemic of nonalcoholic fatty liver disease demonstrates an exponential increase in burden of disease. Hepatology. 2018;67(1):123-33. https://doi. org/10.1002/hep.29466.

12. Patel YA, Gifford EJ, Glass LM, Turner MJ, Han B, Moylan CA, et al. Identifying nonalcoholic fatty liver disease advanced fibrosis in the veterans health administration. Dig Dis Sci. 2018;63(9):2259-66. https://doi. org/10.1007/s10620-018-5123-3.

13. Jaruvongvanich $V$, Wijarnpreecha $K$, Ungprasert P. The utility of NAFLD fibrosis score for prediction of mortality among patients with nonalcoholic fatty liver disease: a systematic review and meta-analysis of cohort study. Clin Res Hepatol Gastroenterol. 2017;41(6):629-34. https://doi. org/10.1016/j.clinre.2017.03.010

14. Bertot LC, Jeffrey GP, Wallace M, MacQuillan G, Garas G, Ching HL, Adams LA. Nonalcoholic fatty liver disease-related cirrhosis is commonly unrecognized and associated with hepatocellular carcinoma. Hepatol Commun. 2017:1(1):53-60. https://doi.org/10.1002/hep4.1018.

15. Hester D, Golabi P, Paik J, Younossi I, Mishra A, Younossi ZM. Among medicare patients with hepatocellular carcinoma, non-alcoholic fatty liver disease is the most common etiology and cause of mortality. J Clin Gastroenterol. 2019. https://doi.org/10.1097/MCG.0000000000001172.

16. Younossi, Z., Stepanova, M., Ong, J. P., Jacobson, I. M., Bugianesi, E., Duseja, A., et al. (2019). Nonalcoholic steatohepatitis is the fastest growing cause of hepatocellular carcinoma in liver transplant candidates. Clin Gastroenterol Hepatol 17(4), 748-755e3. https://doi.org/https://doi.org/10.1016/j. cgh.2018.05.057

17. Tavakoli H, Robinson A, Liu B, Bhuket T, Younossi Z, Saab S, et al. Cirrhosis patients with nonalcoholic steatohepatitis are significantly less likely to receive surveillance for hepatocellular carcinoma. Dig Dis Sci. 2017;62(8):2174-81. https://doi.org/10.1007/s10620-017-4595-X.

18. Singal AG, Tiro J, Li X, Adams-Huet B, Chubak J. Hepatocellular carcinoma surveillance among patients with cirrhosis in a population-based integrated health care delivery system. J Clin Gastroenterol. 2017;51(7):650-5. https://doi.org/10.1097/MCG.0000000000000708.

19. Jun TW, Yeh ML, Yang JD, Chen VL, Nguyen P, Giama NH, et al. More advanced disease and worse survival in cryptogenic compared to viral hepatocellular carcinoma. Liver Int. 2018;38(5):895-902. https://doi. org/10.1111/liv.13613.

20. Tapper EB, Hunink MG, Afdhal NH, Lai M, Sengupta N. Cost-effectiveness analysis: risk stratification of nonalcoholic fatty liver disease (NAFLD) by the primary care physician using the NAFLD fibrosis score. PLOS ONE. 2016;11(2):e0147237. https://doi.org/10.1371/journal.pone.0147237.

21. Srivastava A, Gailer R, Tanwar S, Trembling P, Parkes J, Rodger A, et al. Prospective evaluation of a primary care referral pathway for patients with non-alcoholic fatty liver disease. J Hepatol. 2019;71(2):371-8. https:// doi.org/10.1016/j.jhep.2019.03.033. 
22. Townsend SA, Newsome PN. Review article: new treatments in nonalcoholic fatty liver disease. Aliment Pharmacol Ther. 2017. https://doi. org/10.1111/apt.14210

23. Haigh L, Bremner S, Houghton D, Henderson E, Avery L, Hardy T, et al. Barriers and facilitators to mediterranean diet adoption by patients with nonalcoholic fatty liver disease in Northern Europe. Clin Gastroenterol Hepatol. 2019;17(7):1364-1371.e3. https://doi.org/10.1016/j. cgh.2018.10.044.

24. Romero-Gómez M, Zelber-Sagi S, Trenell M. Treatment of NAFLD with diet, physical activity and exercise. J Hepatol. 2017. https://doi. org/10.1016/j.jhep.2017.05.016.

25. Katsagoni CN, Georgoulis M, Papatheodoridis GV, Panagiotakos DB, Kontogianni MD. Effects of lifestyle interventions on clinical characteristics of patients with non-alcoholic fatty liver disease: A meta-analysis. Metab Clin Exp. 2017. https://doi.org/10.1016/j.metabol.2016.12.006.

26. Kenneally S, Sier JH, Moore JB. Efficacy of dietary and physical activity intervention in non-alcoholic fatty liver disease: a systematic review. BM Open Gastroenterol. 2017;4(1):e000139. https://doi.org/10.1136/bmjga st-2017-000139.
27. Davis JPE, Henry ZH, Argo CK, Northup PG. Relationship of physician counseling to weight loss among patients with nonalcoholic fatty liver disease: an observational cohort study using National Health and Education Survey Data. Clin Liver Dis (Hoboken). 2019;14(4):156-60. https://doi. org/10.1002/cld.832.

28. Sargeant JA, Gray LJ, Bodicoat DH, Willis SA, Stensel DJ, Nimmo MA, et al. The effect of exercise training on intrahepatic triglyceride and hepatic insulin sensitivity: a systematic review and meta-analysis. Obes Rev. 2018;19(10):1446-59. https://doi.org/10.1111/obr.12719.

29. Lam B, Kurzke K, Younossi Z. The clinical and economic burden of nonalcoholic steatohepatitis. Curr Hepatol Rep. 2018;17(4):345-9. https://doi. org/10.1007/s11901-018-0423-9.

\section{Publisher's Note}

Springer Nature remains neutral with regard to jurisdictional claims in published maps and institutional affiliations.
Ready to submit your research? Choose BMC and benefit from:

- fast, convenient online submission

- thorough peer review by experienced researchers in your field

- rapid publication on acceptance

- support for research data, including large and complex data types

- gold Open Access which fosters wider collaboration and increased citations

- maximum visibility for your research: over $100 \mathrm{M}$ website views per year

At BMC, research is always in progress.

Learn more biomedcentral.com/submissions 